\title{
Modelado sísmico en un medio con atenuación usando la ecuación de onda fraccionaria: Difusión-Onda
}

\author{
Leidy Y. Medina (UdeM, UdP), Francisco H. Cabrera (UdP), Cesar A. Arias (ITM, UdeA), Carlos C. Piedrahita (UdeM)
}

Copyright 2016, SBGf - Sociedade Brasileira de Geofísica

Este texto foi preparado para a apresentação no VII Simpósio Brasileiro de Geofísica, Ouro Preto, 25 a 27 de outubro de 2016. Seu conteúdo foi revisado pelo Comitê Técnico do VII SimBGf mas não necessariamente representa a opinião da SBGf ou de seus associados. É proibida a reprodução total ou parcial deste material para propósitos comerciais sem prévia autorização da SBGf.

\section{Resumen}

En la exploración sísmica para la búsqueda de hidrocarburos es importante obtener imágenes con buena aproximación que muestren las estructuras del subsuelo con el fin de ubicar adecuadamente los yacimientos. Las imágenes de las estructuras ubicadas bajo zonas con saturación de gas, pueden ser de baja calidad debido a que las ondas sísmicas que se generan de forma controlada, en la superficie, pierden energía allí y por tanto no logran iluminar bien tales regiones.

En la actualidad se trabaja en técnicas que puedan mejorar la calidad de la imagen sísmica compensando la energía perdida por estos efectos de atenuación desde los mismos modelos de propagación.

Este trabajo aborda este problema mediante el uso de ecuaciones con derivadas fraccionarias las cuales pueden describir la propagación de ondas con atenuación.

\section{Abstract}

In seismic exploration it is important to obtain good seismic images of the structure of the earth to find possible reservoirs. Seismic images of geological structures that are beneath gas saturated zones can be of poor quality, due to the fact that seismic waves that are generated by the sources, loss energy there. Therefore, some regions are poorly illuminated. Currently, several techniques that can improve the seismic images are being used, correcting the energy losses due to attenuation. This can be accomplished, for example, using appropriate models of seismic propagation.

This work addresses this problem using the concept of fractional derivatives, which can describe the effect of wave attenuation.

Keywords: Fractional derivates, attenuation, modelling, stability.

\section{Introducción}

La exploración sísmica demanda técnicas sofisticadas que puedan aplicarse a cualquier medio complejo: medios con fracturas, y otras heterogeneidades.

En particular, en la propagación de ondas sísmicas se producen fenómenos como, atenuación ya sea producida por fenómenos elásticos, como expansión geométrica, multi-trayectorias, scatering o por fenómenos inelásticos(energía se pierde en forma de calor) como la fricción interna y el flujo de fluidos que se encuentra en yacimientos de hidrocarburos de gas, que ocasionan una pérdida significativa en la fuerza de la señal y el ancho de banda, un ejemplo de este efecto ocurre en los campos Valhall y Sleipner(Campos de la petrolera Británica BP), que se encuentran en trampas de gas(Dvorkin, 2006).

Los eventos reflejados encima y por debajo de las regiones con saturaciones de gas, producen una pérdida de las altas frecuencias y disminución de la amplitud, causando que el pulso se deforme.

Para simular con mayor precisión la propagación de ondas sísmicas, se debe tener en cuenta algunos parámetros físicos que describen el tipo de material(medio), en particular, en este trabajo se tendrá en cuenta los efectos de la atenuación con el fin de obtener una mejor aproximación de la imagen del subsuelo para su debida interpretación.

\section{Marco Teórico}

El cálculo fraccionario o integración y derivación de orden arbitrario, tiene su origen a partir de algunas especulaciones de Leibniz, cuando discutía el significado de la derivada $1 / 2$ en una carta a L'Hopital. Leibniz escribió que su resultado era "Una aparente paradoja de la cual algún día se obtendrían consecuencias útiles". Los problemas y áreas del conocimiento en los que se ha aplicado el cálculo fraccionario son numerosos, entre ellos las ecuaciones diferenciales, teoría de probabilidad, teoría de la viscoelasticidad, etc., en particular la ecuación diferencial fraccionaria Difusión-Onda que interpola el fenómeno difusivo y propagatorio (Podlubny, 1999).

Algunas definiciones que se pueden encontrar en la literatura son:

\section{Derivada de Grünwald-Letnikov}

Una de las definiciones de la derivada de GrünwaldLetnikov está dada por la siguiente expresión, la cual es 
una aproximación en diferencias centradas de primer orden:

$$
\frac{\partial^{v} f(t)}{\partial \mathrm{t}^{v}} \sim \frac{1}{h^{v}} \sum_{j=0}^{J}(-1)^{j}\left(\begin{array}{l}
v \\
j
\end{array}\right) f(t-j h)
$$

Donde $v$ es el orden de la derivada, $h$ es el paso de tiempo y $J=t / h-1$.

Algunos ejemplos de la derivada fraccionaria son los siguientes:

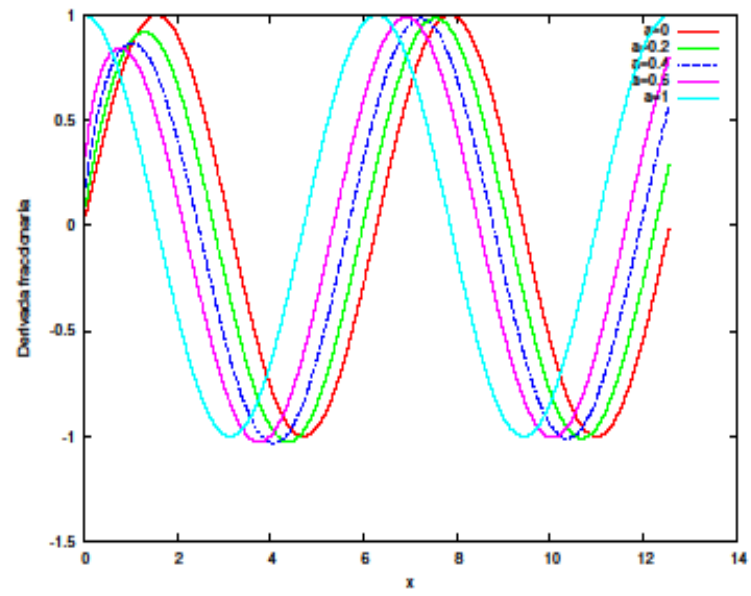

Figura 1: Derivada fraccionaria de la función $f(x)=\sin x$ para valores de la derivada entre 0 y 1 , donde a es el orden de la derivada fraccionaria.

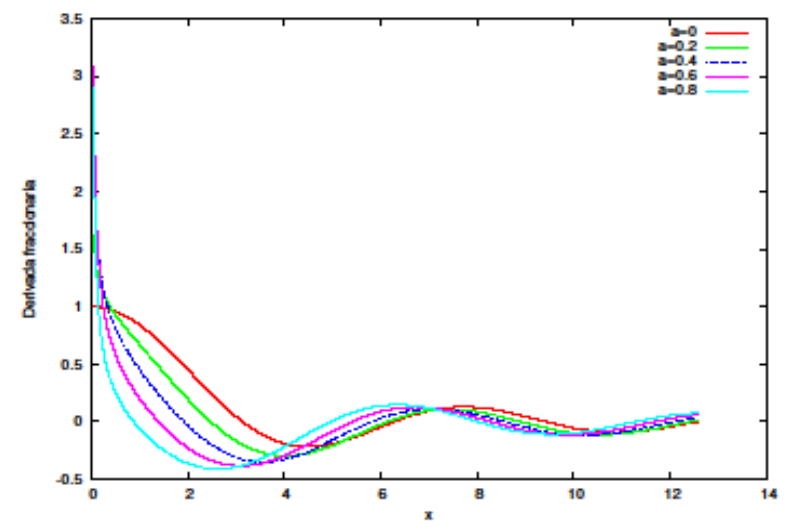

Figura 2: Derivada fraccionaria de la función $f(x)=\frac{\sin x}{x}$ para valores de la derivada entre 0 y 1 , donde a es el orden de la derivada fraccionaria.

\section{Derivada de Caputo}

Otra definición de la derivada fraccionaria es la derivada de Caputo, la cual es operativamente mejor para resolver problemas de valores iniciales. (Podlubny, 1999).

$$
D_{C}^{\alpha} f(t)=\left\{\begin{array}{c}
\frac{1}{\Gamma(m-\alpha)} \int_{0}^{t} \frac{f^{(m)}(\tau)}{(t-\tau)^{\alpha+1-m}} d \tau \\
\frac{d^{m}}{d t^{m}} f(t), \quad \alpha=m
\end{array}\right.
$$

\section{Atenuación}

Varios fenómenos de la propagación de ondas sísmicas en medios complejos (medios anisótropos, medios heterogéneos, medios fracturados.) en particular los medios poroelásticos, las ondas que se propagan en estos medios dependiendo del tipo de material y del tipo de fluido que llenas poros.

La atenuación reúne los efectos de absorción anelástica (en forma de calor), el cual expresa el decaimiento de la amplitud en la propagación de las ondas. La disipación de energía es medida como la energía disipada entre la energía pico almacenada en un ciclo.

Existen varios modelos para medir la disipación de energía uno de ellos es:

\section{Modelo de Kjartansson's}

Este modelo fue propuesto por Kjartansson's donde define el factor de calidad como (Kjartansson's, E. 1979):

$$
Q \approx \frac{1}{2}\left[\cot \left(\frac{\pi}{2} \gamma\right)-\tan \left(\frac{\pi}{2} \gamma\right)\right]
$$

Este modelo es independiente de la frecuencia, por ello se refiere a un modelo de $Q$ constante. Para valores pequeños de $\gamma$, la expresión se reduce:

$$
\gamma \approx \frac{2}{\pi} \tan ^{-1}\left(\frac{1}{Q}\right)
$$

\section{Ecuación de Difusión-Onda}

Una manera de modelar el comportamiento de materiales viscoelásticos son los modelos mecánicos que son descritos por ecuaciones diferenciales de orden arbitrario, en particular la ecuación de difusión-onda. Esta ecuación modela fenómenos, que dependen del valor de la derivada fraccionaria, un fenómeno difusivo y un fenómeno propagatorio. En esta ecuación se puede tener en cuenta las pérdidas de energía de manera natural; es decir que con la ecuación de difusión-onda se puede hacer modelado o migración teniendo en cuenta el llamado factor de calidad.

\section{Solución numérica de la ecuación de difusión-Onda}

En la propagación de las ondas sísmicas en un medio, se modela una ecuación de onda, en este caso se considerará una ecuación diferencial fraccionaria: la ecuación de Difusión-Onda para un medio visco acústico propuesta por Carcione (Carcione $\mathrm{J} \mathrm{M}$, Cavallini F, Mainardi F and Hanyga A. (2002)):

$$
v_{0}^{2} \cos ^{2}\left(\frac{\pi \gamma}{2}\right) \omega_{0}^{-2 \gamma} \nabla^{2} P=\partial_{t}^{2-2 \gamma} P
$$

donde $\gamma=\frac{1}{\pi} \tan ^{-1}\left(\frac{1}{Q}\right), 0 \leq \gamma<\frac{1}{2}$.

La ecuación diferencial fraccionaria descrita en (5) interpola dos fenómenos de la física, el fenómeno difusivo que se cumple cuando el valor $\gamma=\frac{1}{2}$ y el fenómeno propagatorio si $\gamma=0$, el tipo de fenómeno que 
se obtiene depende del orden de la derivada y este orden está relacionado con el factor de calidad Q: Si $Q<<1$ el resultado es un fenómeno más difusivo y si $Q>>1$ el fenómeno es más propagatorio con pérdidas.

Se va a solucionar numéricamente la ecuación (5) por el método numérico de diferencias finitas.

Aproximando el campo de presión por medio del polinomio de Taylor, obteniendo así el operador de segundo orden en diferencias finitas:

$$
\frac{\partial^{2} P}{\partial x^{2}} \approx \frac{P_{i+1, j}^{n}-2 P_{i, j}^{n}+P_{i-1, j}^{n}}{\Delta x^{2}}
$$

Análogamente para $z$

$$
\frac{\partial^{2} P}{\partial z^{2}} \approx \frac{P_{i, j+1}^{n}-2 P_{i, j}^{n}+P_{i, j-1}^{n}}{\Delta z^{2}}
$$

Donde $\Delta x y \Delta z$ son los pasos en la malla y $\Delta t$ es el paso en tiempo.

Reemplazando las expresiones (1), (6) y (7) en la expresión (5), y teniendo en cuenta $\Delta h=\Delta x=\Delta z$ :

$$
\begin{array}{r}
P_{i, j}^{n+1} \approx \Delta t^{2-2 \gamma} v_{0}^{2} \cos ^{2}\left(\frac{\pi \gamma}{2}\right) \omega_{0}^{-2 \gamma}\left[\frac{P_{i+1, j}^{n}-2 P_{i, j}^{n}+P_{i-1, j}^{n}}{\Delta h^{2}}+\right. \\
\left.\frac{P_{i+1, j}^{n}-2 P_{i, j}^{n}+P_{i-1, j}^{n}}{\Delta h^{2}}\right]-\sum_{k=1}^{n} c_{k}^{2-2 \gamma} P_{i, j}^{n-k+1}
\end{array}
$$

donde

$$
c_{k}=(-1)^{k}\left(\begin{array}{c}
2-2 \gamma \\
k
\end{array}\right)
$$

son los

pesos

correspondientes a la derivada fraccionaria, se obtiene la ecuación en diferencias.

\section{Estabilidad numérica}

La estabilidad está relacionada con garantizar que un algoritmo no acumule errores de redondeo. Para analizar la estabilidad del esquema numérico descrito en la expresión (8) se considera que los errores son de la forma armónica, expresados de la siguiente manera:

$$
\varepsilon_{i, j}^{n}=\xi_{n} e^{I \kappa_{x} \Delta x+I \kappa_{z} \Delta z}
$$

donde $I=\sqrt{-1}$, es la unidad imaginaria y $\kappa_{x}, \kappa_{z}$ son las componentes del vector de onda. Sustituyendo (9) en el esquema numérico (8) y realizando operaciones algebraicas se obtiene que la condición de estabilidad para la ecuación en diferencias descrito en la ecuación (8) es la siguiente:

$$
\frac{2 v_{0}^{2} \Delta t^{2-2 \gamma}}{\Delta h^{2}} \leq \frac{4^{-\gamma}}{2 \cos ^{2}\left(\frac{\pi \gamma}{2}\right) \omega_{0}^{-2 \gamma}}
$$

donde $\gamma=\frac{1}{\pi} \tan ^{-1}\left(\frac{1}{Q}\right), 0 \leq \gamma<\frac{1}{2}$. Además si $\gamma=0$ entonces la ecuación (10) representa la condición de Courant para un esquema en diferencias finitas de segundo orden en tiempo y espacio de la ecuación de Onda.

\section{Resultados}

Modelo 1: El primer experimento numérico que se realizó es en una estructura que de un solo medio homogéneo con velocidad constante. Se obtuvo el campo de onda de un medio visco acústico homogéneo para diferentes valores de $\mathrm{Q}$ (factor de calidad). Las dimensiones de este modelo son: $300(\mathrm{~m})$ de longitud por $300(\mathrm{~m})$ de profundidad, la fuente está ubicada en la posición $(x s, Z s)=(250,150)$, la velocidad de la onda $\mathrm{P}$, es de $V p=2500 \mathrm{~m} / \mathrm{s}$. La atenuación es uno de los factores que causa las pérdida la amplitud y de la fase del frente de onda, dichas pérdidas se observa una difusión en el frente de onda en la región donde se encuentra la trampa de gas.

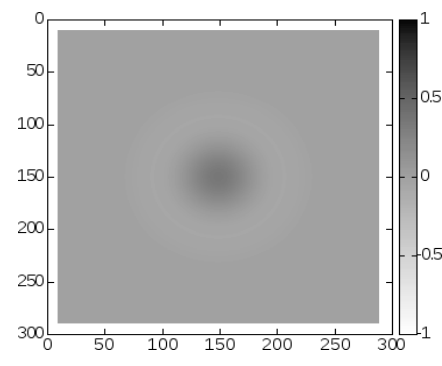

a) $Q=1$

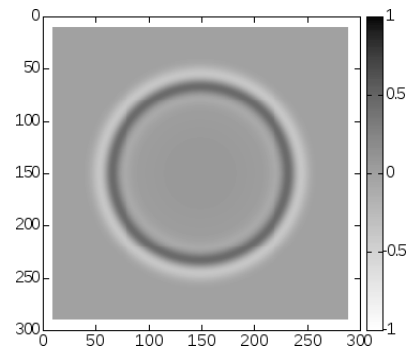

c) $Q=10$

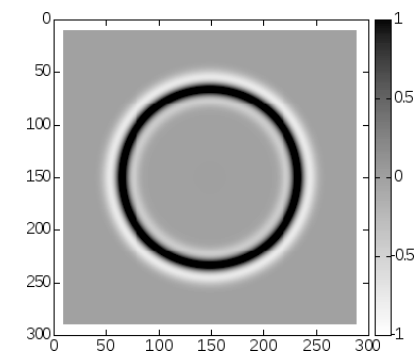

e) $Q=50$

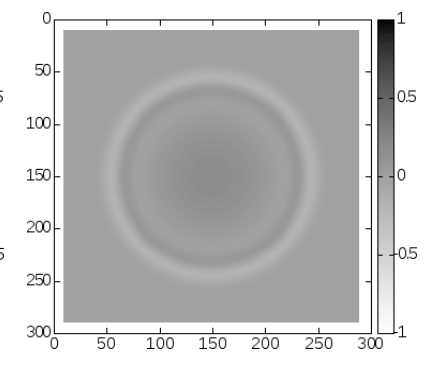

b) $Q=5$

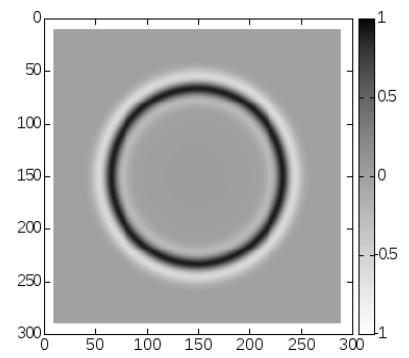

d) $Q=20$

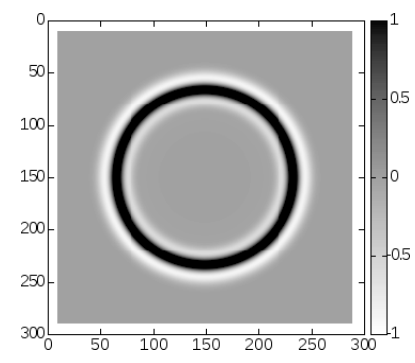

f) $Q=500$
Figura 3: Instantáneas para diferentes valores de $Q p$

Modelo 2. El siguiente modelo es la estructura de un anticlinal, en este modelo se visualiza una trampa de gas, que es una de los causas de las pérdida la amplitud y de la fase. Los datos sintéticos de la estructura del anticlinal fueron generados con software Seismic Unix(Su) con el comando Trimodel. 


\begin{tabular}{|c|c|c|}
\hline Medio & $\mathbf{V}_{\mathbf{p}}(\mathbf{m} / \mathbf{s})$ & $\mathbf{Q}_{\mathbf{p}}$ \\
\hline 1 & 2600 & 50 \\
\hline 2 & 3200 & 80 \\
\hline 3 & 4000 & 100 \\
\hline 4 & 5200 & 180 \\
\hline 5 & 3650 & 17 \\
\hline 6 & 4300 & 45 \\
\hline 7 & 6000 & 280 \\
\hline
\end{tabular}

Tabla 1: Valores de la velocidad y valor del factor de calidad para la onda $P$.

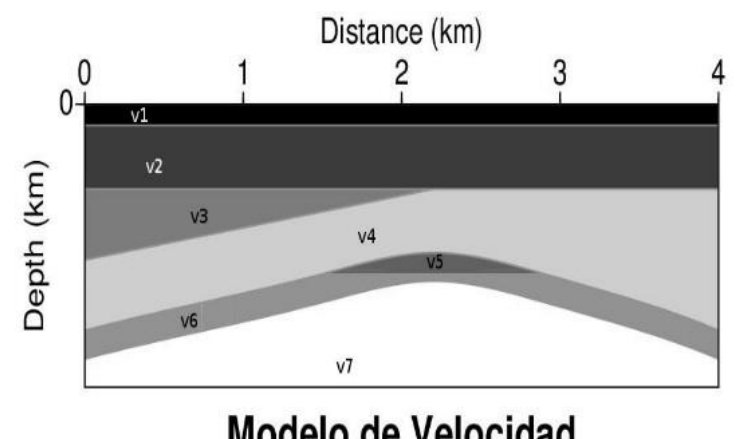

Figura 4: Modelo de velocidades de una estructura anticlinal

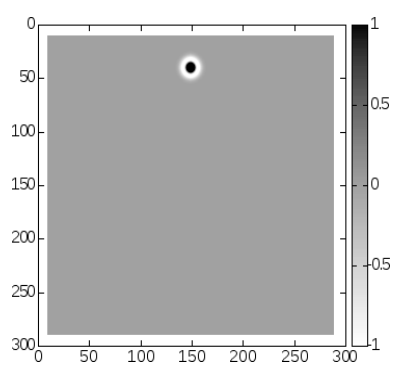

a) $t=0 s$

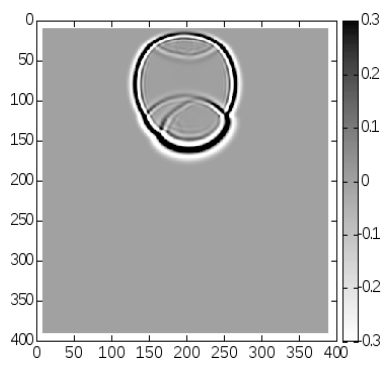

c) $t=0.032 \mathrm{~s}$

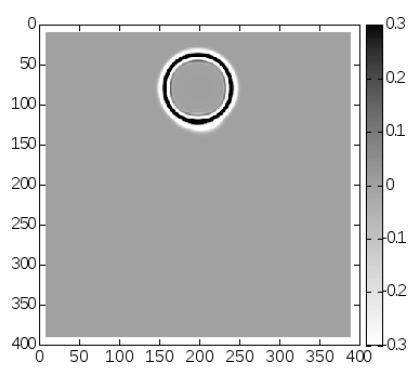

b) $t=0.02 \mathrm{~s}$

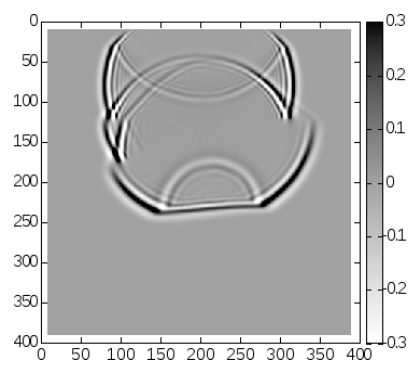

d) $t=0.057 \mathrm{~s}$
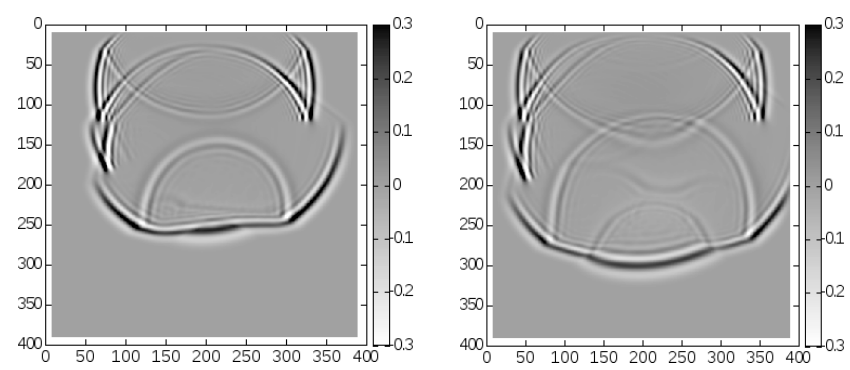
e) $\mathrm{t}=0.066 \mathrm{~s}$
f) $t=0.077 \mathrm{~s}$

Figura 5: Instantáneas en diferentes tiempo de la propagación

En la figura (5) se pueden observar la propagación en una estructura anticlinal en diferentes tiempos; se puede visualizar que cuando la onda sísmica cambia de litología o pasa de un medio a otro, esta pierde energía, en particular en el medio 5, el cual tiene un bajo valor de $Q$.

\section{Conclusiones}

Se implementó un código de modelado sísmico basado en derivadas fraccionarias con el fin de estudiar la propagación de ondas sísmicas en medios con atenuación, el cual se puede aplicar a yacimientos no convencionales para mejorar la estimación del factor de calidad (Q).

A través de ejemplos numéricos se mostró la transición que existe de un fenómeno difusivo a un fenómeno propagatorio mediante el uso de las derivadas fraccionarias.

Se logró determinar una expresión para la estabilidad del esquema numérico en diferencias de la ecuación de difusión-onda, la cual es comparable con las condiciones ya establecidas, por ejemplo si $\gamma=0$ se obtiene la condición de estabilidad para la ecuación de onda 2-D, llamada condición de COURANT.

\section{Agradecimientos}

Este trabajo fue financiado por ECOPETROL y COLCIENCIAS, mediante el proyecto: "Migración sísmica pre-apilado en profundidad por extrapolación del campo de onda utilizando computación de alto desempeño para datos masivos en zonas complejas".

\section{Referencias}

Aki, Keiiti and Richards, Paul G. (1980), Quantitative seismology, University Science Books, ed.2,

Alford R and Kelly, K. (1974). Accuracy of finite-difference modeling of acoustic wave propagation. Geophysics 39, 834-842. 
Carcione J M, Cavallini F, Mainardi $F$ and Hanyga A. (2002) Time domain modeling of constant $Q$ seismic wave using fractional derivatives, Pure and Applied Geophys, 159: 1719-1736.

Carcione J M.(2007) Wave fields in Real Media.Wave propagation in Anisotropic, Anelastic, porous and electromagnetic media, Elsevier $\mathbf{3 8}$.

Dvorkin, J. P., and G. Mavko (2006). Modeling attenuation in reservoir and nonreservoir rock: The Leading Edge, 25(2), 194-197.

Dutta G, Kai Lu, XinWang and Schuster G. T (2013).

Attenuation compensation in least-squares reverse time migration using the visco-acoustic wave equation.

University of Science and Technology.

Gazdag, J. (1978). Wave equation migration with the phase shift method, Geophysics, 73-2: 1342-1351

Kjartansson, E.(1979) Constant-Q wave propagation and attenuation. Journal of Geophysical Research, 84, B9: 4737-4748.

Mainardi, F (2010). Fractional calculus and waves in linear viscoelasticity: An Introduction to Mathematical Models. Imperial College Press.

Podlubny, Igor (1999). Fractional diferential Equations: An introduction to fractional derivates, Fractional Diferential Equations, to methods of their solution and some of their Applicacions. Mathematics in science and engineerin, Academic Press, San Diego, Calif USA. 198.

Zhu, T., and J. M. Harris (2014). Modeling acoustic wave propagation in heterogeneous attenuating media using decoupled fractional Laplacians. Geophysics, 79-3: S165S174.

Medina L, Cabrera F, Arias C, Piedrahita C, (2016). Manuscrito Tesis de Maestría: "Modelado sísmico en un medio con atenuación usando la ecuación de onda: difusión-onda" en preparación. Departamento de Ciencias Básicas Universidad de Medellín. 